\title{
Spontaneous response tendencies in noncontingent trials of a matching-to-position task in rats: Consequences for learning the matching and nonmatching task contingencies
}

\author{
ARJAN BLOKI.AND \\ University of Limburg, Maastricht, The Netherlands \\ and \\ STEPHEN B. DUNNETT \\ University of Cambridge, Cambridge, England
}

\begin{abstract}
Previous studies have indicated that rats show differences in their acquisition of two closely related delayed response tasks in two-lever operant chambers: delayed matching to position and delayed nonmatching to position. In order to analyze these differences, separate groups of rats were exposed to alternative sets of noncontingent pretraining, during which their responses did not influence the reward outcome, prior to training on the matching contingency in Experiment 1 or the nonmatching contingency in Experiment 2. The alternative pretraining conditions included: fixed or random presentation of the sample lever, different levels of reinforcement of responses to the sample lever $(0 \%$ or $50 \%)$, and different levels of reinforcement of responses to the choice lever $(50 \%$ or $100 \%$ ). When the sample lever was always on the same side, all rats adopted progressively strong side biases in responding to the choice levers, some to the same side as that of the sample lever and others to the opposite side, with approximately equal frequency. When the sample lever was presented randomly and not reinforced, rats again initially adopted strong side biases, the strength of which declined modestly with repeated training. Only when both the side of the sample lever and the probability of reinforcement of the sample response was $50 \%$ did the rats adopt a spontaneous tendency to match the sample and choice responses. This resulted in enhanced learning of the matching-toposition contingency and retarded acquisition of the nonmatching contingency. The results indicate that both spontaneous response tendencies and previous experience can influence rats' acquisition of conditional discrimination tasks widely used in the study of short-term memory processes.
\end{abstract}

Delayed matching and nonmatching tasks have provided a powerful technique for the analysis of short-term memory in a variety of different species. Humans, monkeys and pigeons have all been widely studied, with 3-dimensional "junk" objects or computer-generated patterns used as visual stimuli in delayed matching- and nonmatching-to-sample tests (DMTS and DNMTS, respectively) (Blough, 1959; D'Amato, 1973; Jarrard \& Moise, 1971; Mishkin, 1982; Oscar-Berman \& Bonner, 1989; Pontecorvo \& Evans, 1985; Roberts, 1980; Sahakian et al., 1988; Sahakian et al., 1993; Zola-Morgan,

This research was supported by grants from the Mental Health Foundation and the Netherlands Organization for Scientific Research. A.B.'s current address is the Institute for Neurobiology, Department of Gerontopharmacology, Troponwerke, Berlinerstrasse 156, D-51063 Cologne, Germany. S.B.D. is in the MRC Cambridge Centre for Brain Repair, University of Cambridge. Correspondence should be addressed to S. B. Dunnett, Department of Experimental Psychology, Downing Street, Cambridge CB2 3EB, England.
Squire, \& Amaral, 1989). In particular, the need to generate large numbers of sample trials in order to obtain accurate estimates of performance at different delay intervals has led to the widespread adoption of automated tests run under computer control in many of these studies (Blough, 1959; D'Amato, 1973; Jarrard \& Moise, 1971; Oscar-Berman \& Bonner, 1989; Pontecorvo \& Evans, 1985; Roberts, 1980; Sahakian et al., 1988; Sahakian et al., 1993). Although rats can also be trained to discriminate physical objects or distinctive patterned environments in choice boxes and Y-mazes (Aggleton, 1985; Rothblat \& Hayes, 1987), the visual modality is not predominant in this species, and it has proved difficult to train rats to use visual stimuli in operant versions of DMTS and DNMTS tasks (Dunnett, 1985; ZolaMorgan et al., 1989). This problem has at least partially been resolved, however, by requiring rats to use spatial sample and choice information in a variety of delayed response, and delayed matching- and nonmatching-toposition tasks (DMTP and DNMTP, respectively) (Bier- 
ley \& Kesner, 1980; Dunnett, 1985, 1993; van Haaren \& van Hest, 1989; Wallace, Steinert, Scobie, \& Spear, 1980 ). Although a variety of maze learning procedures are also available for measuring spatial working memory in rats, such as the radial or T-mazes (Jarrard, 1993; Markowska, Olton, Murray, \& Gaffan, 1989; Olton, Becker, \& Handelmann, 1979), DMTP and DNMTP have the distinct advantage of providing automated collection of data from the very large numbers of trials that must be run to obtain accurate estimates of forgetting functions across a series of delay intervals (Dunnett, 1993).

In the DMTP/DNMTP task, rats are trained in an operant chamber with two independently retractable levers and a central food well (Dunnett, 1993). Each trial is divided into a sample phase, during which the rat must respond to a left or right lever presented at random; a delay phase of variable duration, during which the rat is required to maintain responding at the central food well panel; and a choice phase, during which the rat must make a choice between the two levers. In the DMTP version of the task, the rat is rewarded for responding to the same lever as that presented during the sample phase, whereas in the DNMTP version, it must respond to the opposite lever. Rats readily learn these two alternative sets of contingencies, and the tasks have now been used by a number of laboratories for investigating the parameters of normal short-term memory (the slope of the delay-performance providing a direct index of the rate of forgetting) (Dunnett \& Martel, 1990; Sahgal, 1987); the changes associated with aging, lesions, or ischaemia (Aggleton, Hunt, \& Shaw, 1990; Aggleton, Keith, \& Sahgal, 1991; Dunnett, Evenden, \& Iversen, 1988; Dunnett, Rogers, \& Jones, 1989; Rogers et al., 1992; Sahgal, Keith, Lloyd, et al., 1990); and the effects of alternative drug or neural transplant treatments (Deacon, 1991; Dunnett, Badman, Rogers, Evenden, \& Iversen, 1988; Dunnett \& Martel, 1990; Sahgal, Keith, \& Lloyd, 1990a, 1990b; van Hest, Stroet, van Haaren, \& Feenstra, 1990).

The matching and nonmatching contingencies involved in the two primary versions of the DMTP/ DNMTP task are logically symmetrical, and they were initially considered to be rather similar. Indeed, in a number of studies, the two versions of the task have been used to provide replications of basic phenomena, such as delay-dependent deficits in aged rats or the absence of an effect owing to quisqualic acid lesions (in contrast to ibotenic acid lesions) of the basal forebrain (Dunnett, Evenden, \& Iversen, 1988; Dunnett et al., 1989; Sahgal et al., 1990b). Nevertheless, it has become apparent that the two versions of the task have important differences.

First, the delay-dependent deficits in aged animals appeared to develop at an earlier age in the DMTP version of the task than in the DNMTP version, although this apparent difference may have reflected little more than variability among the rather small number of animals in the middle-aged groups (Dunnett, Evenden, \& Iversen, 1988).

Second, it appears that normal young animals initially acquire the matching and nonmatching contingencies in different ways (Dunnett, 1993; Dunnett, Evenden, \& Iversen, 1988). Thus, in the nonmatching version of the task, each individual animal shows gradual improvement from day to day, yielding smooth mean acquisition curves for the whole group, with relatively low variance at all time points. Conversely, in the matching version of the task, a few animals learn the task very rapidly whereas others become locked into position habits, always responding to just one lever whichever the side of the sample. As the position habit is broken from day to day, each animal then switches relatively promptly to adopting a matching response. The result of this pattern of responding by individual animals is that the mean acquisition curve in the matching task can again appear relatively smooth; but it has a higher variance at intermediate time points and in fact approximates to the summation of a series of independent step functions. Whereas correction procedures to break position habits are not required when rats are trained in the nonmatching contingency, they are routinely used for the matching contingency.

Third, particular lesions and drugs have been found to dissociate acquisition (but not performance) of the two contingencies. For example, lesions of the basal forebrain disrupt both the initial acquisition of the nonmatching contingency and the reversal of an animal pretrained on the matching task to the nonmatching contingency, but have no detectable effect on the converse set of circumstances: neither the initial acquisition of the matching contingency nor the reversal of an animal pretrained on the nonmatching task to the matching contingency are affected (Dunnett et al., 1989). In addition, certain drugs, such as vasopressin, can have different effects on the two tasks, improving performance on the matching contingency but impairing performance in the nonmatching contingency (Sahgal et al., 1990b).

These results suggested that the rats' spontaneous response biases might influence the ease of learning the two versions of the task, as well as the effects of different structural or pharmacological manipulations in the brain. However, spontaneous response tendencies have not previously been determined in this task. A number of alternatives may be proposed (Dunnett et al., 1989). First, since rats alternate spontaneously in a variety of situations such as the conventional T-maze (Dember \& Fowler, 1958), the nonmatching task may be easier to learn. However, the tendency to alternate in mazes is most plausibly related to strategies for optimal foraging (Kamil \& Roitblat, 1985), in which animals do not immediately return to places in which food stores have been depleted, and a similar principle may not be applicable in operant tasks, which are governed instead by principles of reinforcement (Ferster \& Skinner, 1957). Specifically, in two-lever operant chambers, rats tend to repeat a previously rewarded response but to switch to a different response when not rewarded (so called "winstay/lose-shift" behavior) (Evenden \& Robbins, 1982). However, the status of the sample response in the standard version of the DMTP and DNMTP task is uncer- 
tain. On the one hand, the rats do not receive a food pellet in response to the sample leverpress (the "lose" condition). On the other hand, the panel light, which is associated with reward in the choice phase and so will acquire secondary reinforcing properties, is also turned on when the rat presses the sample lever (in order to cue nosepoking during the delay phase) and so might be expected to yield the "win" condition (Dunnett et al., 1989). A third factor is that it is conceivable that some elements of the delayed matching contingency could be facilitated by the formation of a Pavlovian association between the sample and the (same) choice response, whereas the adoption of a different response in the nonmatching version of the task requires that the animal learn a conditional decision rule (Gaffan, 1987).

Initial training in the DMTP/DNMTP tasks begins in all cases without scheduled delays (i.e., MTP/NMTP) prior to training with progressively lengthened delay intervals (Dunnett, 1993). The present experiments were designed first to identify spontaneous behavioral tendencies in a noncontingent version of the standard MTP/NMTP task and second to investigate whether different noncontingent conditions of reinforcement during pretraining would influence subsequent acquisition of the matching and nonmatching contingencies.

\section{EXPERIMENT 1}

\section{Method}

\section{Subjects}

Sixty young adult female Sprague-Dawley rats were used, weighing $180-200 \mathrm{~g}$ at the start of the experiment. They were housed in groups of 6 rats per cage, under a natural light:dark cycle, with water available ad lib. Behavioral tests were conducted between 0900 and $1500 \mathrm{~h}$, during which the rats were subjected to a fooddeprivation regime; they were given 10-12 g dry laboratory chow at the end of each day's testing, to maintain body weight at approximately $90 \%$ of the free-feeding level.

\section{Apparatus}

Tests were conducted in a bank of 10 operant chambers (Campden Instruments, London) under the on-line cont rol of two Acorn Archimedes microcomputers. Each chamber was equipped with two retractable levers spaced $7.5 \mathrm{~cm}$ on either side of a central food well that was accessed by pushing back a hinged Perspex panel. A microswitch detected whenever the rat inserted its nose into the food well by pressing back the panel. A houselight was positioned in the center of the ceiling to illuminate the chamber, and a panel light was positioned to illuminate the food well from be- hind the panel. A food dispenser delivered single 45-mg sucrose pellets (BioServe, USA) to the food well.

\section{Procedure}

Pretraining. The rats were initially trained in $30-\mathrm{min}$ sessions over 4 consecutive days to associate the click of the food dispenser and the illumination of the panel light with delivery of food pellets to the food well and to collect them by pressing back the panel. They were then trained over 3 further days on a discrete-trial continuous reinforcement schedule. On each trial, one lever, chosen at random, was extended into the chamber. As soon as the rat pressed it, the lever was retracted, the panel light was turned on, and a food pellet was delivered. As soon as the rat pressed back the panel to collect the pellet, the panel light was turned off, and a 5-sec intertrial interval preceded the next presentation of one lever or the other. Within 3 days, all rats had acquired the simple leverpress response reliably (more than 80 responses per 30 -min session).

Noncontingent training trials. The rats were then trained on alternative noncontingent versions of the standard MTP and NMTP training schedule. Each trial was structured as follows. First, depending on the particular schedule, one of the two levers was selected as the sample and was extended into the chamber. When the rat pressed the sample lever, the lever was retracted and the panel light was turned on. Next, when the rat pressed the panel, the panel light was turned off and both levers were inserted into the chamber for the rat to make a choice. As soon as the rat pressed one lever or the other, the two levers were retracted. On some or all trials, depending on the schedule, a food pellet was presented following the choice response, in which case the panel light was turned on until the rat made a panel press to collect the pellet. On other, nonreinforced, trials, the houselight was switched off for $5 \mathrm{sec}$. Then, in either case, a 15 -sec intertrial interval preceded the next trial.

The rats were trained according to one of five different schedules of (noncontingent) reinforcement (summarized in Table 1):

Group FIX 1.0. The same lever was always used as the sample (the left lever for half the rats and the right lever for the other half); the sample response was never reinforced; the choice response was reinforced on every trial.

Group FIX 0.5 . The same lever was always used as the sample (the left lever for half the rats and the right lever for the other half); the sample response was never reinforced; the choice response was reinforced with a $50 \%$ probability, irrespective of the animals' choice.

Group RAN 1.0. The sample lever was selected at random between the left and right levers (50\% probability of either); the sample response was never reinforced; the choice response was reinforced on every trial.

Group RAN 0.5. The sample lever was selected at random between the left and right levers ( $50 \%$ probability of either); the sample response was never reinforced; the choice response was reinforced with a $50 \%$ probability, irrespective of the animals' choice.

Group REINF S/C. The sample lever was selected at random between the left and right levers ( $50 \%$ probability of either); the

Table 1

Experimental Training Conditions

\begin{tabular}{lllcr}
\hline & & & \multicolumn{2}{c}{ Reinforced Responses } \\
\cline { 5 - 5 } \multicolumn{1}{c}{ Group } & $n$ & \multicolumn{1}{c}{ Sample Lever } & Sample & Choice \\
\hline FIX 1.0 & 10 & one side only & no & $100 \%$ \\
FIX 0.5 & 10 & one side only & no & $50 \%$ \\
RAN 1.0 & 10 & left or right, at random & no & $100 \%$ \\
RAN 0.5 & 10 & left or right, at random & no & $50 \%$ \\
REINF S/C & 20 & left or right, at random & $50 \%$ & $50 \%$ \\
\hline
\end{tabular}

Note-For definition of groups, see Experiment 1, Method. 
sample response was reinforced with a $50 \%$ probability, irrespective of the side of the sample; the choice response was also reinforced with a $50 \%$ probability, irrespective of the animals' choice.

Thus the noncontingent trials were structured identically to the first stage (i.e., with zero delays) of the training procedure for the MTP and NMTP tasks, other than that the conventional reinforcement contingencies were made constant or random. All rats were trained in the noncontingent schedules in 30 -min daily sessions over 15 days.

Two measures were collected to characterize the spontaneous response tendencies of the rats during the noncontingent training:

$$
\text { percent matching }=100 \times \frac{\text { (choice responses to sample lever) }}{\text { (total responses) }}
$$$$
\text { response bias }=\frac{\text { (choice responses to preferred lever) }}{\text { (total responses) }}
$$

For analysis, the daily data were collapsed into five blocks of 3-day sessions. Differences between groups were analyzed with a two-factor analysis of variance (group $\times$ session) with repeated measures on the second factor. Duncan's multiple-range test was used to evaluate differences between groups post hoc. In addition, the performance of the REINF S/C group was analyzed separately for trials on which the sample response was reinforced and trials on which the sample response was not reinforced.

Acquisition of the MTP task. The rats were finally trained on the MTP task (i.e., with zero scheduled delays) for another fifteen 30-min sessions. The structure of the trials was similar to that used during the noncontingent training, but now with contingent reinforcement of the choice response. Specifically, on each trial, the sample lever was selected at random, the response to the sample lever was never reinforced, and both levers were presented for the choice response, which was reinforced if and only if the rat pressed

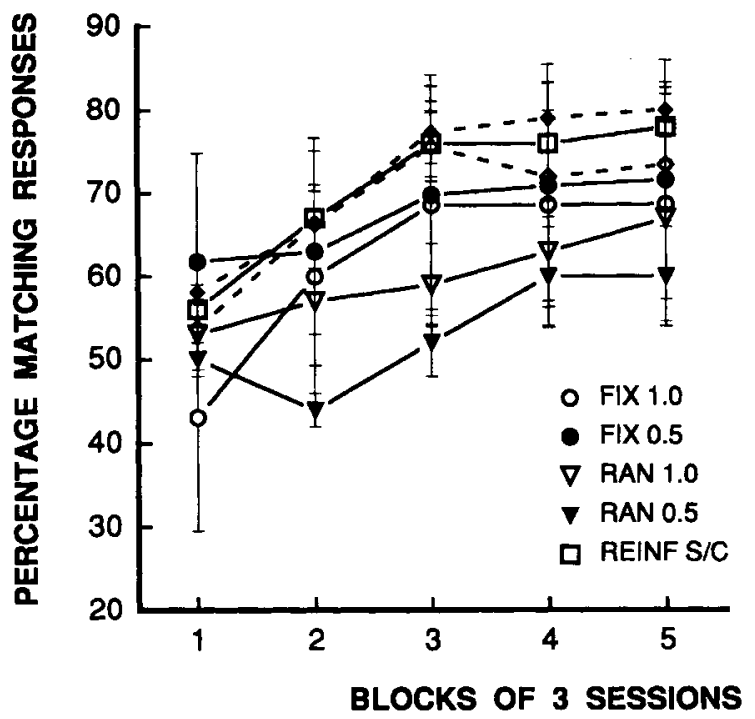

Figure 1. Percentage of trials $(M \pm S E M)$ on which the animals' choice responses matched (i.e., were on the same lever as) the sample lever, over consecutive 3-day blocks of training sessions in the noncontingent version of the task. The training conditions in the five groups are as specified in Table 1. The dashed lines represent the data for group REINF S/C subdivided into trials on which the sample was reinforced (small filled diamonds) and on which it was not reinforced (small open diamonds).

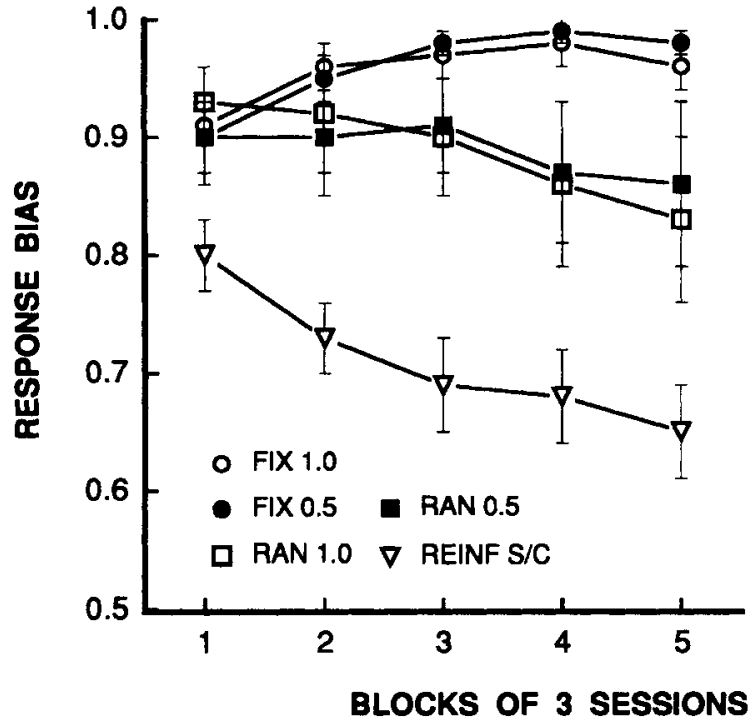

Figure 2. Response bias $(M \pm S E M)$, defined as the proportion of trials on which responses were made by each animal to the side on which it made the higher number of choice responses, of the five groups of animals over consecutive 3-day blocks of training sessions in the noncontingent version of the task. The training conditions in the five groups are as specified in Table 1.

the same lever as that presented during the sample. The intertrial interval was $5 \mathrm{sec}$. The acquisition of the contingent matching task was compared in terms of the percentage of correct matching responses between the five different groups.

\section{Results}

\section{Noncontingent Training Trials}

The percent matching responses of each group across sessions is shown in Figure 1. With repeated testing in the various noncontingent versions of the task, all groups showed a progressively increasing tendency to select the choice response that was the same as the sample lever. The percentage of matching responses increased during the course of training in all groups to a similar extent [sessions, $F(4,220)=12.95, p<.01$; groups, $F(4,55)=$ 0.87, n.s.; groups $\times$ sessions, $F(16,220)=0.97$, n.s.]. Subsequent analysis of performance by the REINF S/C group indicated no significant differences between the percentages of matching responses on trials when the sample had and had not been reinforced [dashed lines in Figure $1 ; F(1,38)=0.93$, n.s.].

The development of response bias in the different training conditions is shown in Figure 2. All groups manifested marked response biases, with a tendency to respond to one lever in preference to the other in the choice phase. However, the four groups in which the sample response was never reinforced showed a higher level of response bias than did the REINF S/C group, in which there was $50 \%$ reinforcement of the sample [main group effect, $F(4,55)=11.95, p<.01]$. Moreover, whereas the rats in the two conditions when the sample was always on the same side (FIX 0.5 and FIX 1.0) showed progres- 


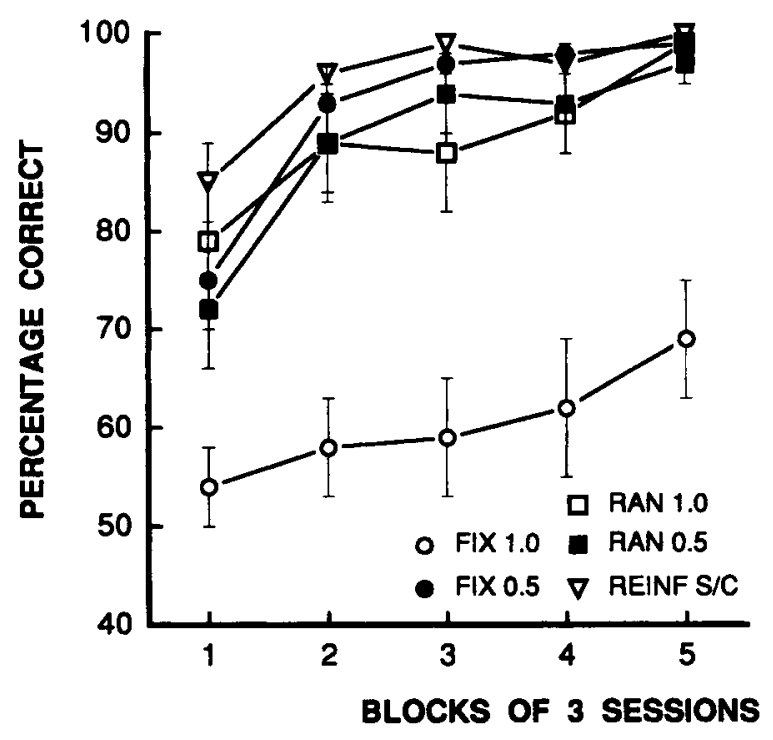

Figure 3. Pencentage correct responses $(M \pm S E M)$ of the five groups of animals over consecutive 3-day blocks of training sessions when the rats were switched to the contingent matching-to-position task in the first experiment. The training conditions in the five groups are as specified in Table 1.

sively increased response bias, the other three groups in which the side of the sample varied (RAN 0.5 , RAN 1.0 and REINF S/C) all showed a modest decline in their response biases [group $\times$ session interaction, $F(16,220)=$ $2.26, p<.01]$.

\section{Acquisition of the Matching Contingency}

As is shown in Figure 3, the percent correct responses in the matching task increased significantly in the course of training [sessions, $F(4,220)=40.25, p<.001$ ]. However, whereas four of the groups rapidly learned the task up to a high level of accuracy within 3-5 days of testing, the FIX 1.0 showed very poor performance [groups, $F(4,55)=21.33, p<.001]$. Inspection of the performance of individual cases indicated that six of the 10 rats in the FIX 1.0 group still showed a strong side bias (>98\% of response to one side) even during the last 3 days of testing.

\section{EXPERIMENT 2}

In the first experiment, we found a clear difference between the FIX 1.0 group and the other four experimental groups in their acquisition of the matching contingency of the MTP task. Most remarkable was the difference between the FIX 1.0 and FIX 0.5 groups, both of which were trained on the same schedule of reinforcement except that the probability of reinforcement differed in the choice phase, and which developed similar degrees of spontaneous matching and response bias. We therefore evaluated in a second experiment whether the pretraining on the FIX 1.0 and FIX 0.5 schedules had similar consequences for learning the nonmatching contingency. In addition, the REINF S/C condition was also included in the second experiment because, although the differences did not reach significance, this group developed the highest tendency to match in the noncontingent trials of the first experiment.

\section{Method}

\section{Subjects}

Thirty young adult female Sprague-Dawley rats were used, weighing $150-180 \mathrm{~g}$ at the start of the experiment. Housing, lighting and food-deprivation conditions were the same as in Experiment 1 .

\section{Procedure}

The rats were randomly divided into three groups $(n=10$ in each), pretrained, and then tested on the FIX 0.5 , FIX 1.0 , or REINF S/C noncontingent reinforcement schedules, in the same apparatus and under identical training conditions as those in Experiment 1 . After 15 sessions of noncontingent training, all rats received a further 15 sessions of training on the nonmatching contingency. Specifically, on each trial the sample lever was selected at random, the response to the sample lever was never reinforced, and both levers were presented for the choice response, which was reinforced if and only if the rat pressed the lever on the side opposite to that presented during the sample. The intertrial interval was $5 \mathrm{sec}$. The acquisition of the contingent nonmatching task was compared between groups in terms of the percentage of correct matching responses between the three different groups.

\section{Results}

\section{Noncontingent Training Trials}

The pattern of results in Experiment 2 was almost identical to that observed in Experiment 1, for which it provides a replication. Thus, the tendency to match choice responses to the sample lever increased in the course of training [sessions, $F(4,108)=4.01, p<.01$ ] and did not differ between the three groups [groups, $F(2,27)=0.55$; groups $\times$ sessions, $F(8,108)=0.48$; both n.s.]. Also similar was the fact that the response bias was significantly lower in the REINF S/C group than in the two FIX groups [groups, $F(2,27)=5.81, p<$ 01 ], and whereas the REINF S/C groups showed progressively lower response bias with repeated testing, the bias of the two FIX groups increased [groups $\times$ sessions, $F(8,108) \cdot=2.56, p<.02]$.

\section{Acquisition of the Nonmatching Contingency}

As shown in Figure 4, the percent correct responses in the nonmatching task increased significantly in the course of training in all groups [sessions, $F(4,108)=$ $58.78, p<.001]$. There were again significant differences between groups [groups, $F(2,27)=4.18, p<.01$; groups $\times$ sessions, $F(8,108)=2.36, p<.05$ ]; but their magnitude was smaller, and the pattern was very different from that observed in Experiment 1. The FIX 0.5 group actually showed the highest percent correct nonmatching scores overall, and the REINF S/C the lowest, with the FIX 1.0 group intermediate and differing from neither. The lower nonmatching scores of the REINF $\mathrm{S} / \mathrm{C}$ were most apparent during the first few days of test- 


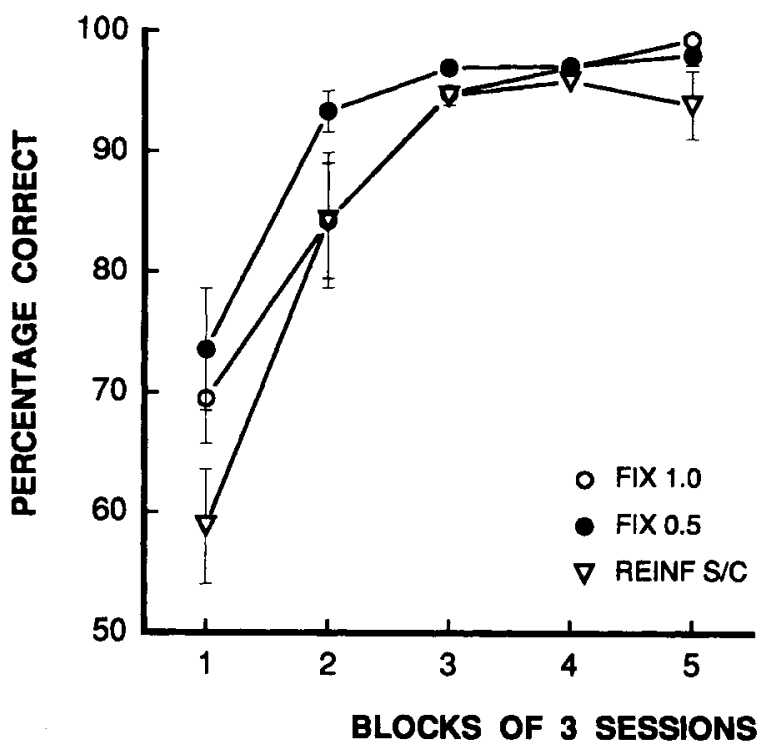

Figure 4. Percentage correct responses $(M \pm S E M)$ of the three groups of animals in the second experiment over consecutive 3-day blocks of training sessions when the rats were switched to the contingent nonmatching-to-position task. The training conditions in the three groups are as specified in relevant rows of Table 1.

ing, whereas all groups converged on a high level of performance ( $>90 \%$ correct) within 6-9 days of training, and the groups did not differ significantly thereafter.

\section{DISCUSSION}

The present results confirm previous observations that when rats have multiple choices available to them in an operant chamber they adopt distinctive response strategies. The pattern of spontaneous bias appeared to be influenced more by regularities in the structure of the trials than by the probabilities of subsequent reinforcement, which was not contingent upon the choice response, and under some circumstances was found to have a profound influence on their subsequent acquisition of tasks based upon contingent reinforcement.

\section{Spontaneous Choice Behavior}

The most clear-cut feature of the results was that under most circumstances the rats exhibited individual distinctive side biases. This was true of all rats from the outset of training, but was further influenced (whether strengthened or weakened) by the schedule employed in subsequent training.

Side bias and matching are not independent and need to be considered in the context of each schedule. This is simplest in the two FIX conditions in which only one lever was ever used as the sample. In this situation, if each individual animal had a bias toward matching, the sample lever would always be the preferred lever, and the response bias measure (Equation 2) would be identical to the matching measure (Equation 1)-apart from the fact that one is expressed as a percentage. Comparison of the FIX 0.5 and FIX 1.0 curves in Figures 1 and 2 indicates that this is not so. Rather, as both groups converge on a bias of 1.0 , with low standard errors, the percentage of matching responses converges on approximately $70 \%$, but with considerably higher variability between animals. This represents the fact that all animals are developing rigid side biases in the choice phase, with 7 out of the 10 animals in each group responding consistently to the same lever as the constant sample, whereas the other 3 animals in each group consistently respond to the opposite lever. In the second experiment, 8 out of 20 animals in the FIX condition responded to the same lever and 12 responded to the opposite lever. Thus, the rats appear to have had a strong tendency to adopt the strategy of always responding on the same side, which is not readily explained in terms of either matching or nonmatching tendencies per se. The strong tendency of the animals in the FIX condition (in which the structure of the trials and side of the sample lever remained constant throughout training) to adopt a stereotyped pattern of responding was not influenced by whether the choice was reinforced on every trial or only on $50 \%$ of the trials.

The situation is a little more complicated in the two RAN groups. These animals initially exhibited a similar degree of response bias as the FIX groups, but the bias declined with subsequent testing and became considerably more variable. Since the sample lever is presented on either the left or the right side, with equal probability, a strong side bias will produce a matching score that converges on $50 \%$. This was true of most animals at the outset of training and showed only moderate change in the course of training. As in the case of the FIX rats, neither the stereotyped patterns of responding nor the tendency of the animals in the RAN condition to make matching rather than alternating choices was influenced by whether the choice was reinforced on every trial or only on $50 \%$ of the trials.

Of particular interest is the condition provided by the REINF S/C group. The structure of the trials and the contingencies in the choice phase were identical with those for the RAN 0.5 group, from which the REINF $\mathrm{S} / \mathrm{C}$ group differed only in that it also received food pellet rewards to pressing the sample lever on $50 \%$ of the trials. This noncontingent reinforcement of the sample lever reduced the side bias considerably and was lower than that observed in any of the other groups. The moderate initial level of bias declined further with continued testing, and all rats responded to both levers in the choice phase. The rats trained under the REINF S/C condition adopted a significant tendency toward matching which was the highest overall level of all the groups (at approximately $75 \%$ ). Of particular importance for the interpretation of these results is the fact that the REINF S/C rats showed an identical pattern of choice matching on trials when the sample was reinforced as on trials when it was not reinforced. This suggests that in the particular task structure employed here, the tendency of the animals to 
match is not readily explained in terms of a winstay/lose-shift hypothesis (Evenden \& Robbins, 1982), since they are making "stay" responses with a similar probability whether the sample responses are rewarded ("win") or not ("lose"). Similarly, a tendency to alternate, as has been described in T-maze tasks (Dember \& Fowler, 1958), does not appear to apply in the present operant situation. Rather, it is plausible that the unpredictability of the outcome of the animals' responses to the sample lever in the REINF S/C group may have the effect of increasing levels of attention, surprise, or arousal, any of which may be associated with behavioral flexibility and disrupt a strong side bias (Mackintosh, 1974; Yerkes \& Dodson, 1908).

\section{Acquisition of the Matching and Nonmatching Contingencies}

The present experiments were not primarily designed to investigate spontaneous choice behavior per se, but to understand better how intrinsic tendencies of the rats to make certain choices spontaneously might influence subsequent learning of the DMTP and DNMTP tasks, and what the effects of pharmacological manipulations or experimental lesions on acquisition of those tasks might be.

In the conventional procedure used to train rats in the DMTP task (Dunnett, 1993), the animals progress directly from the continuous reinforcement pretraining to the zero-delay (MTP) condition of the delayed matching contingency. Under such circumstances, a proportion of the animals adopt a strong fixed position habit, which can substantially retard training, in the absence of a correction procedure. The present pretraining exposure to alternative conditions exaggerated these effects in the FIX 1.0 group but reduced them in the others. Thus, all animals exposed to the FIX 1.0 conditions maintained their strong position habits throughout acquisition of the MTP task, and the majority of animals failed to show any deviation from that inefficient strategy even after more than 2 weeks of further training. By contrast, perhaps the more remarkable outcome was the rapidity of learning of the matching and nonmatching contingencies to a high level in the other four groups. In spite of the strong response biases that were allowed to develop over 15 days in the noncontingent phase of the present experiments, all the animals in these groups were able to detect when the contingencies changed, adapt their choice behavior accordingly, and learn the task rapidly.

The FIX 1.0 group was distinguished by the absence of any variability in the noncontingent task contingencies: every sample was presented on the same side, the sample response was never reinforced, and the choice response was always reinforced. Since each trial was totally predictable, the rats not only developed strong and rigid response biases, but their attention to the redundant stimuli and the outcomes of their responses would have declined, which would have compromised the animals' subsequent ability to learn to use those stimuli as the sample for matching once they became predictive of rein- forcement (Mackintosh, 1974). Although the FIX 0.5 group developed an equally strong side bias, the outcomes of the rats' choice responses remained unpredictable, and this appeared to have been sufficient to maintain the animals' attention and to enable their detection of a change in contingencies once the task was switched to acquisition of the matching task. Similarly, although every choice response was reinforced in the RAN 1.0 group, the side of the sample remained unpredictable, and this also appears to have been sufficient to maintain efficient subsequent learning.

More difficult to explain is why this failure by the FIX 1.0 group alone applied in the acquisition of the matching contingency but not in acquisition of the nonmatching contingency. The differences in acquisition of the two versions of the contingent task in the FIX 1.0 group were clear, consistent between rats, and very highly significant, whereas the rats that underwent the other noncontingent training conditions all appeared to be able to learn both tasks without difficulty. The difference between the matching and nonmatching tasks in the FIX 1.0 group indicates that the two sets of contingencies can be learned in different ways or are dependent on different underlying processes. One possibility is that the noncontingent phase of training may influence the establishment of classically conditioned associations between the sample and choice phases of the task. We have previously speculated that the matching version of the contingent task is potentially more subject to Pavlovian conditioning, whereas the nonmatching task is more likely to require use of a conditional discrimination rule (Dunnett et al., 1989). In the present context, particularly strong associations might be expected to have formed between the sample lever and the choice response lever in rats of the FIX 1.0 group, in whom virtually every noncontingent trial involved reinforcement of the same sequence of responses. In all other pretraining conditions, either many sample trials were not reinforced, or both sample levers were reinforced to a similar degree. The resulting Pavlovian associations would have been strongest and have had the greatest disturbance on acquisition of the contingent task in the FIX 1.0 group. Moreover, this would be likely to have had a greater influence on acquisition of the matching than of the nonmatching version of the task, exactly as observed.

The only other clear differences between the two experiments were that the REINF $\mathrm{S} / \mathrm{C}$ rats exhibited a higher level of performance on the matching task than on the nonmatching task during the first 6 days of training, which is almost certainly attributable to their spontaneous tendency to matching at a relatively high level in the preceding noncontingent trials, and so they started learning the conditional matching task from a considerably higher baseline than that in the conditional nonmatching task (i.e., approx. $70 \%$ vs. $30 \%$ ).

\section{Conclusions}

Rats learn the contingent operant task against a baseline of a number of spontaneous response biases and in- 
trinsic habits. The initial spontaneous response tendencies that an animal brings to a new task environment include strong biases to respond on just one side and, dependent upon experience, a tendency to match responses. These spontaneous biases not only interact with each other from the outset, but can also change in strength with experience and take on the form of habits. Not only will these spontaneous tendencies influence subsequent learning of the actual task contingencies, but the establishment of particular response habits through experience can substantially modify the rate of learning the primary matching or nonmatching task contingencies. Moreover, the distinctive effects of the different noncontingent training conditions on rats' abilities to acquire the matching and nonmatching contingencies of the MTP/NMTP task corroborates the view that these apparently symmetrical contingencies involve fundamentally different processes that are learned in different ways. Consequently, account must be taken of the attentional and associational processes that underlie rats' acquisition and performance of the DMTP and DNMTP tasks when they are used for the analysis of processes affecting short-term memory.

\section{REFERENCES}

Aggleton, J. P. (1985). One-trial object recognition by rats. Quarterly Journal of Experimental Psychology, 37B, 279-294.

Aggleton, J. P., Hunt, P. R., \& SHAw, C. (1990). The effects of mammillary body and combined amygdalar-fornix lesions on tests of delayed non-matching-to-sample in the rat. Behavioural Brain Research, 40, 145-157.

Aggleton, J. P., Keith, A. B., \& Sahgal, A. (1991). Both fornix and anterior thalamic, but not mammillary, lesions disrupt delayed nonmatching-to-position memory in rats. Behavioural Brain Research, 44, 151-161

BIERLEY, R. A., \& KeSNER, R. P. (1980). Short-term memory: The role of the midbrain reticular formation. Journal of Comparative \& Physiological Psychology, 94, 519-529.

BLough, D. S. (1959). Delayed matching in the pigeon. Journal of the Experimental Analysis of Behavior, 2, 151-160.

D'Amato, M. R. (1973). Delayed matching and short-term memory in monkeys. Psychology of Learning \& Motivation, 7, 227-269.

DEACON, R. M. J. (1991). Pharmacological studies of a rat spatial delayed nonmatch-to-sample task as an animal model of dementia. Drug Development Research, 24, 67-79.

Dember, W. N., \& Fowler, H. (1958). Spontaneous alternation behavior. Psychological Bulletin, 55, 412-428.

DUNNETT, S. B. (1985). Comparative effects of cholinergic drugs and lesions of nucleus basalis or fimbria-fornix on delayed matching in rats. Psychopharmacology, 87, 357-363.

DUNNETT, S. B. (1993). Operant delayed matching and non-matching to position in rats. In A. Sahgal (Ed.), Behavioural neuroscience: $A$ technical approach (pp. 123-136). Oxford, U.K.: IRL Press.

Dunnett, S. B., Badman, F., Rogers, D. C., Evenden, J. L., \& IVERSEN, S. D. (1988). Cholinergic grafts in the neocortex or hippocampus of aged rats-reduction of delay-dependent deficits in the delayed non-matching to position task. Experimental Neurology, $102,57-64$.

Dunnett, S. B., Evenden, J. L., \& Iversen, S. D. (1988). Delaydependent short-term memory deficits in aged rats. Psychopharmacology, 96, 174-180.

Dunnett, S. B., \& Martel, F. L. (1990). Proactive interference effects on short-term memory in rats: 1 . Basic parameters and drug effects. Behavioral Neuroscience, 104, 655-665.
Dunnett, S. B., Rogers, D. C., \& Jones, G. H. (1989). Effects of nucleus basalis magnocellularis lesions in rats on delayed matching and non-matching to position tasks: Disruption of conditional discrimination learning but not of short-term memory, European Journal of Neuroscience, 1, 395-406.

Evenden, J. L., \& RobBins, T. W. (1982). Win-stay behaviour in the rat. Quarterly Journal of Experimental Psychology, 36B, 1-26.

FERSTER, C. B., \& SKINNER, B. F. (1957). Schedules of reinforcement. New York: Appleton-Century-Crofts.

GAFFAN, D. (1987). Amnesia, personal memory, and the hippocampus: Experimental neuropsychological studies in monkeys. In S. M. Stahl, S. D. Iversen, \& E. C. Goodman (Eds.), Cognitive neurochemistry (pp. 21-36). Oxford: Oxford University Press.

JARRARD, L. E. (1993). On the role of the hippocampus in learning and memory in the rat. Behavioral \& Neural Biology, 60, 9-26.

JARRARD, L. E., \& MoISE, S. L. (1971). Short-term memory in the monkey. In L. E. Jarrard (Ed.), Cognitive processes of nonhuman primates (pp. 1-24). New York: Academic Press.

Kamil, A. C., \& RoItblat, H. L. (1985). The ecology of foraging behavior: Implications for animal learning and memory. Annual Review of Psychology, 36, 141-169.

Mackintosh, N. J. (1974). The psychology of animal learning. New York: Academic Press.

Markowska, A. L., Olton, D. S., Murray, E. A., \& Gaffan, D. (1989). A comparative analysis of the role of fornix and cingulate cortex in memory: Rats. Experimental Brain Research, 74, 187-201.

Mishkin, M. (1982). A memory system in the monkey. Philosophical Transactions of the Royal Society of London: Series B, 298, 85-95.

Olton, D. S., Becker, J. T., \& HandelmanN, G. E. (1979). Hippocampus, space and memory. Behavioral \& Brain Sciences, 3, 313365.

OsCar-Berman, M., \& BonNer, R. T. (1989). Nonmatching- (oddity) and delayed nonmatching-to-sample performance in aging, alcoholic, and alcoholic Korsakoff individuals. Psychobiology, 17, 424430.

Pontecorvo, M. J., \& Evans, H. L. (1985). Effects of aniracetam on delayed matching-to-sample performance of monkeys and pigeons. Pharmacology, Biochemistry \& Behavior, 22, 745-752.

ROBERTS, W. A. (1980). Distribution of trials and intertrial retention in delayed matching to sample with pigeons. Journal of Experimental Psychology: Animal Behavior Processes, 6, 217-237.

Rogers, D. C., Wright, P. W., Roberts, J. C., Reavill, C., Rothaul, A. L., \& Hunter, A. J. (1992). Photothrombotic lesions of the frontal cortex impair the performance of the delayed non-matching to position task by rats. Behavioural Brain Research, 49, 231-235.

RothBlat, L. A., \& HAYES, L. L. (1987). Short-term object recognition memory in the rat: Nonmatching with trial-unique junk stimuli. Behavioral Neuroscience, 101, 587-590.

Sahakian, B. J., Morris, R. G., Evenden, J. L., Heald, A., Levy, R., PhILPOT, M., \& Robrins, T. W. (1988). A comparative study of visuospatial memory and learning in Alzheimer-type dementia and Parkinson's disease. Brain, 111, 695-718.

Sahakian, B. J., Owen, A. M., Morant, N. J., Eagger, S. A., Boddington, S., Crayton, L., Crockford, H. A., Crooks, M., HILL, K. \& LEVY, R. (1993). Further analysis of the cognitive effects of tetrahydroaminoacridine (THA) in Alzheimer's disease: Assessment of attentional and mnemonic function. Psychopharmacology, 110, 395-401.

SaHgal, A. (1987). Some limitations of indices derived from signal detection theory: Evaluation of an alternative index for measuring bias in memory tasks. Psychopharmacology, 91, 517-520.

Sahgal, A., Keith, A. B., \& Lloyd, S. (1990a). Effects of nicotine, oxotremorine and 9-amino 1,2,3,4-tetrahydroacridine (tacrine) on matching and non-matching to position in rats: No evidence for mnemonic enhancement. Journal of Psychopharmacology, 4, 210 218.

SAHGAL, A., KeIth, A. B., \& LloYd, S. (1990b). Opposing effects of vasopressin on matching versus non-matching to position: Further evidence for response, not memory, modulation. Psychopharmacology, 102, 130-135. 
Sahgal, A., Keith, A. B., Lloyd, S., Kerwin, J. M., Perry, E. K., \& EDWARDSON, J. A. (1990). Memory following cholinergic (NBM) and noradrenergic (DNAB) lesions made singly or in combination: Potentiation of disruption by scopolamine. Pharmacology, Biochemistry \& Behavior. 37, 597-605.

VAN HAaReN, F., \& VAN HEST, A. (1989). Spatial matching and nonmatching in male and female Wistar rats: Effects of delay-interval duration. Animal Learning \& Behavior, 17, 355-360.

van Hest, A., Stroet, J., van Haaren, F., \& Feenstra, M. (1990). Scopolamine differentially disrupts the behavior of male and female $W$ istar rats in a delayed nonmatching to position procedure. Pharmacology, Biochemistry \& Behavior, 35, 903-909.

Wallace, J., Steinert, P. A., Scobie, S. R., \& Spear, N. E. (1980).
Stimulus modality and short-term memory in rats. Animal Learning \& Behavior, $8,10-16$

YERKES, R. M., \& DODSON, J. D. (1908). The relation of strength of a stimulus to rapidity of habit formation. Journal of Comparative \& Neurological Psychology, 18, 458-482.

Zola-Morgan, S., Squire, L. R., \& amaral, D. G. (1989). Lesions of the hippocampal formation but not lesions of the fornix or mammillary nuclei produce long-lasting memory impairment in monkeys. Journal of Neuroscience, 9, 898-913.

(Manuscript received November 16, 1993; revision accepted for publication September 8, 1994.) 